LUNG CANCER

\title{
Oesophageal endoscopic ultrasound with fine needle aspiration improves and simplifies the staging of lung cancer
}

\author{
H Kramer, J W G van Putten, W J Post, H M van Dullemen, A H H Bongaerts, J Pruim, \\ A J H Suurmeijer, T J Klinkenberg, H Groen, H J M Groen
}

Thorax 2004;59:596-601. doi: 10.1136/thx.2003.018028

See end of article for authors' affiliations

Correspondence to: Mr H Kramer, Groningen University Hospital, Department of Pulmonary Diseases, P O Box 30001 9700 RB Groningen, The Netherlands; h.kramer@ int.azg.nl

Received 29 October 2003 Accepted 14 April 2004

\begin{abstract}
Background: Positron emission tomography (PET) is accurate for mediastinal staging of lung cancer but has a moderate positive predictive value, necessitating pathological verification. Endoscopic ultrasonography with fine needle aspiration (EUS-FNA) is a technique for tissue verification of mediastinal and upper retroperitoneal abnormalities. The use of EUS-FNA may decrease the number of surgical procedures and thereby staging costs.

Methods: EUS-FNA was used prospectively for the cytological assessment of mediastinal and/or upper retroperitoneal PET hot spots in patients with suspected lung cancer. Only if EUS-FNA was positive for malignancy was subsequent mediastinoscopy or exploratory thoracotomy cancelled. The cost effectiveness of EUS-FNA was determined.

Results: Of 488 consecutive patients with suspected lung cancer, 81 were enrolled with mediastinal and/ or upper retroperitoneal PET hot spots. EUS-FNA was positive in $50(62 \%)$ patients, negative in six, and inconclusive in 25 . Of the 31 negative or inconclusive patients, 26 underwent surgical staging (resulting in 14 patients with and 12 without mediastinal malignancy), while five patients had mediastinal metastases during follow up. No EUS-FNA related morbidity or mortality was encountered. The accuracy of the decision to proceed to surgery (or not) on the basis of EUS-FNA was $77 \%$ (95\% Cl 68 to 86 ). EUS-FNA detected more mediastinal abnormalities than PET except for the upper mediastinal region. Addition of EUS-FNA to conventional lung cancer staging reduced staging costs by $40 \%$ per patient, mainly due to a decrease in surgical staging procedures.

Conclusion: EUS-FNA can replace more than half of the surgical staging procedures in lung cancer patients with mediastinal and/or upper retroperitoneal PET hot spots, thereby saving $40 \%$ of staging costs.
\end{abstract}

T he prognosis of patients with lung cancer largely depends on the presence of mediastinal and distant metastases. Malignant involvement of mediastinal lymph nodes is a highly significant prognostic factor for survival. ${ }^{2}$ Complete surgical resection of lung cancer is often impossible in patients with mediastinal metastases. Better preoperative staging may limit the number of unnecessary surgical interventions in these patients. ${ }^{3}$ Positron emission tomography (PET) with ${ }^{18}$ F-fluorodeoxyglucose (FDG) has already shown interesting properties in this area. Oesophageal endoscopic ultrasonography with fine needle aspiration (EUS-FNA) is a technique for the assessment of lymph nodes and tumours in previously almost inaccessible areas such as the lower mediastinum and upper retroperitoneum. Incorporation of this tumour verification technique in lung cancer patients may improve staging of the disease.

FDG-PET is more accurate than computed tomography (CT) in detecting mediastinal lymph node metastases, with a negative predictive value of $93-95 \%{ }^{4-6}$ However, a positive predictive value of $74-90 \%$ makes pathological verification of hot spots necessary to avoid patients being denied a possible cure by surgery..$^{4-7}$ Various staging modalities are used for pathological examination of mediastinal hot spots such as mediastinoscopy, mediastinotomy, and exploratory thoracotomy. However, these techniques are invasive and require general anaesthesia and often hospitalisation, and it is difficult to assess the entire mediastinum-especially the posterior and lower parts. Nevertheless, mediastinoscopy is traditionally considered the "gold standard" for establishing mediastinal metastases. ${ }^{8}$

EUS-FNA combines an excellent view into the mediastinum (EUS) with safe and accurate fine needle aspiration (FNA). It is performed in a short outpatient setting without general anaesthesia, and assessment of at least seven of the 13 right and left mediastinal lymph node levels is possible. ${ }^{9}{ }^{10}$ The upper retroperitoneum can also be evaluated, especially the area around the celiac trunk and the left adrenal gland. ${ }^{11}$ The exact role of EUS-FNA in lung cancer staging is not yet defined, and it is not clear whether it can be used with CT and PET to discriminate accurately between resectable and irresectable lung cancer. Establishing mediastinal or upper retroperitoneal metastases with EUS-FNA makes surgical staging redundant and may result in cost savings for the staging process.

A prospective study was undertaken to determine whether EUS-FNA can reduce the number of surgical staging procedures in lung cancer patients with mediastinal and/or upper retroperitoneal PET hot spots, and whether this approach is cost effective.

Abbreviations: $\mathrm{CT}$, computed tomography; EUS, endoscopic ultrasonography; FNA, fine needle aspiration; PET, positron emission tomography 


\section{METHODS}

\section{Patients}

Consecutive patients with suspected or pathologically established lung cancer were eligible if they had mediastinal and/ or upper retroperitoneal PET hot spots without evidence of further distant metastases, if they had a resectable primary tumour as judged by two thoracic surgeons, and if they were medically operable. All patients gave informed consent and the study was approved by the medical ethics committee of the Groningen University Hospital.

Patients were evaluated by history, physical examination, complete blood cell count, renal and liver function tests, chest radiography, bronchoscopy, CT scan of the chest and upper abdomen, and FDG-PET. All test results were presented in a multidisciplinary session where disease stage, tumour resectability, and medical operability were verified before EUS-FNA was performed.

\section{Imaging tests}

CT was performed with contrast enhanced single and multislice scanners (Somatom Sensation 16/Somatom AR, Siemens AG, Forchheim, Germany). It was used to assess the resectability of the primary tumour, to evaluate mediastinal lymph nodes, and to exclude distant metastases. Whole body PET was performed with an ECAT 951/31 or ECAT HR+ scanner (Siemens/CTI, Knoxville, TN, USA) 90 minutes after intravenous injection of a median dose of $390 \mathrm{MBq}$ (range 130-670) FDG. Attenuation correction was obtained on the HR+ camera by transmission scanning with a germanium-68/ gallium-68 rod source using an interleaved protocol. PET was used for the detection of mediastinal and distant metastases. If mediastinal and/or upper retroperitoneal hot spots were detected, PET was considered positive. Patients with hot spots suggesting metastases outside these areas were excluded from the study. A hot spot was defined as a focal increase in FDG uptake compared with the background not explained by physiological uptake. Both CT and PET were used as guidance for EUS-FNA.

\section{EUS-FNA}

EUS was performed with a linear scanning ultrasound endoscope (FG-34UX, Pentax GmbH, Hamburg, Germany) connected to a scanner unit (EUB-525, Hitachi Ultrasound BV, Reeuwijk, the Netherlands) with Doppler flow imaging for the detection of blood vessels. Midazolam 3-5 mg was administered intravenously for conscious sedation with continuous monitoring of pulse rate and peripheral oxygen saturation. The endoscope was introduced into the stomach to scan the left adrenal gland and the area around the celiac trunk. After retraction into the oesophagus, the mediastinum was evaluated by scanning $360^{\circ}$ transaxially at $1-2 \mathrm{~cm}$ intervals upward up to Naruke level 2. The left adrenal gland was considered enlarged if it had lost its normal elliptical, triangular, or "seagull" shape. ${ }^{11}$ Lymph nodes were identified as non-pulsating regions with round, ellipsoid, crescent, or triangular shape. ${ }^{12}$ They were separately punctured with a 22 gauge needle (Echotip, Wilson-Cook Medical Inc, Winston-Salem, NC, USA) using a $10 \mathrm{ml}$ syringe for suction. A cytotechnician evaluated the cellular contents of the specimens on site with a modified Giemsa method. If a specimen was inadequate, additional punctures were performed. Specimens were categorised as positive (tumour cells), negative (lymphoid but no tumour cells), or inconclusive (poor cellularity or unable to perform adequate puncture). Subsequent mediastinoscopy and/or exploratory thoracotomy with mediastinal dissection was performed in all patients except those with a positive EUS-FNA. The mediastinal lymph node map of the American Joint
Committee on Cancer was used to localise abnormalities at PET, EUS-FNA, and mediastinal dissection. ${ }^{2}$

Because EUS-FNA is a new procedure for lung cancer staging, we were interested in the learning curve of two residents. During each session their ability to identify mediastinal lymph nodes was recorded. One of the residents performed EUS-FNA and an experienced pulmonary physician repeated the procedure. The numbers of observed mediastinal lymph nodes of the residents and the pulmonary physician were compared.

\section{Economic analysis}

Economic analysis was performed to assess the cost effectiveness of adding EUS-FNA to the staging algorithm. It was performed from a hospital point of view. All direct medical costs were included until the final lung cancer stage was established. This also included functional tests or additional examinations necessary for the evaluation of the patient's operability. All cost items of EUS-FNA, mediastinoscopy, and exploratory thoracotomy were estimated by expert opinions of pulmonary physicians, thoracic surgeons, anaesthesiologists, and by observations of two hospital economists. Calculations were based on real costs using the 2002 price level. Costs of hospital admissions were calculated from the actual number of admission days to the general ward and intensive care unit against the Dutch unit price for hospital admission days. ${ }^{13}$ Overhead costs were calculated as a $35 \%$ surcharge. Because of the short time horizon of the diagnostic process, discounting for future costs was not applied.

\section{Statistical analysis}

The study was designed to determine prospectively the proportion of lung cancer patients with mediastinal and/or upper retroperitoneal PET hot spots in whom EUS-FNA can diagnose malignancy. Since EUS-FNA can reach at least seven of the 14 (right and left) mediastinal lymph node levels and the upper retroperitoneum, we estimated that about 50\% of the surgical staging procedures could be avoided. Mediastinal PET has a positive predictive value of $67 \%{ }^{6}$ We therefore expected EUS-FNA to be able to identify $50 \%$ of this $67 \%$ as pathologically malignant-that is, $34 \%$ of all PET

\begin{tabular}{|lc|}
\hline Table 1 Characteristics of the 81 patients with \\
suspected or established lung cancer and \\
mediastinal and/or upper retroperitoneal PET \\
hot spots \\
\hline Characteristic & No (\%) \\
\hline Male sex & $54(67)$ \\
ECOG performance score & $12(15)$ \\
0 & $69(85)$ \\
1 & \\
Pathological diagnosis & $20(25)$ \\
Squamous cell carcinoma & $17(21)$ \\
Adenocarcinoma & $28(35)$ \\
Large cell carcinoma & $6(7)$ \\
Small cell carcinoma & $3(4)$ \\
Malignant lymphoma & $1(1)$ \\
Sarcoidosis & $1(1)$ \\
Aspergillosis & $1(1)$ \\
Unknown & $4(5)$ \\
Other & $13(16)$ \\
CT characteristics & \\
Mediastinum and retroperitoneum & \\
normal & \\
Enlarged mediastinal lymph nodes & $56(69)$ \\
Mediastinal and retroperitoneal & $8(10)$ \\
abnormalities & \\
No CT performed & $4(5)$ \\
\hline & \\
& \\
\hline
\end{tabular}



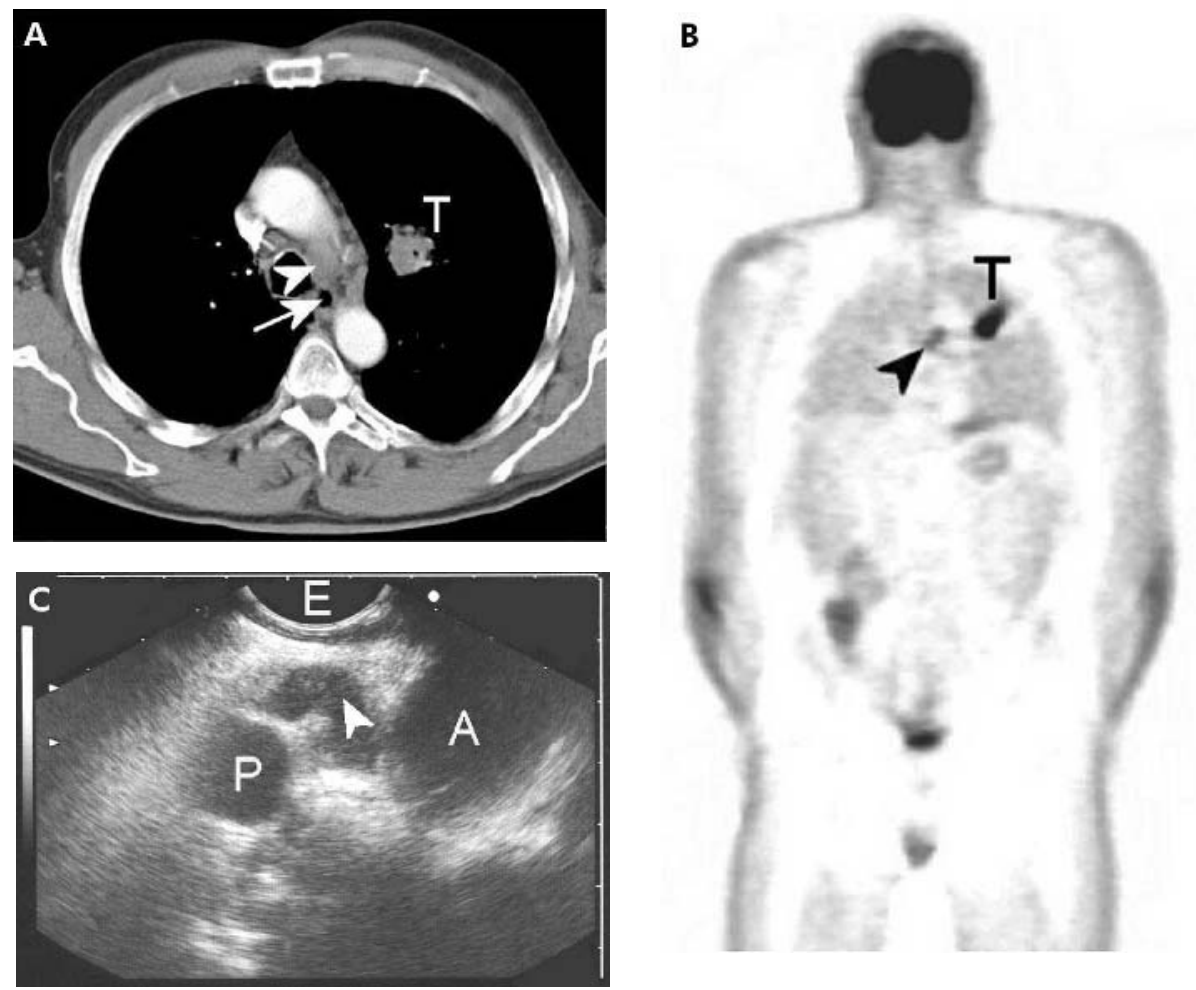

Figure 1 Images of a 54 year old man with squamous cell lung carcinoma. (A) Helical contrast enhanced CT scan (transaxial section) showing a left upper lobe tumour (T) and enlarged mediastinal lymph nodes at Naruke 4-left (arrowhead) next to the oesophagus (arrow). (B) PET scan (coronal section) showing FDG uptake in the tumour (T) and at Naruke 4-left (arrowhead). (C) EUS (sagittal section) showing a hypoechoic lymph node at Naruke 4-left (arrowhead) next to the oesophagus (E). The aortopulmonary window is located between the aortic arch (A) and the left pulmonary artery (P).

positive patients. Eighty patients were needed to estimate this proportion with a precision of $10 \%$.

Statistical analysis was performed with SPSS 10.0 (SPSS Inc, Chicago, IL, USA). Continuous variables are reported as median (range) or mean (SE). Dichotomous variables are reported as percentages with 95\% confidence interval (95\% $\mathrm{CI})$. The accuracy of EUS-FNA was calculated as the accuracy of the decision to proceed to surgical staging (or not) based on EUS-FNA results. For comparisons between groups of categorical variables, Pearson's $\chi^{2}$ test was used. Interobserver agreement for test results was calculated with Cohen's $\kappa$ value. Multiple logistic regression analysis was used to identify ultrasound features predictive for lymph node malignancy. We performed sensitivity analysis of the cost of the staging procedures by varying the major cost elements. ${ }^{14}{ }^{15}$ Reported $p$ values are two sided and $p$ values $<0.05$ were considered significant.

\section{RESULTS}

\section{Patients}

Between January 2001 and March 2003, 488 consecutive patients were screened at the outpatient department, 81 of whom fulfilled all the inclusion criteria and were enrolled in the study (table 1). Their median age was 63 years (range 32$80)$.

\section{Imaging tests}

Images from a typical diagnostic procedure are shown in fig 1. Sixty four patients (79\%) patients had mediastinal and/or upper retroperitoneal abnormalities on the CT scan. Interobserver agreement ( $\kappa$ value) for the detection of enlarged lymph nodes and left adrenal enlargement on CT scanning was 0.76 (95\% CI 0.66 to 0.86$)$. All patients had
Table 2 Number of patients with abnormalities at PET and EUS per level, and the number patients in whom FNA was performed per level

\begin{tabular}{lccc} 
& $\begin{array}{l}\text { PET } \\
\text { hot spot }\end{array}$ & $\begin{array}{l}\text { EUS } \\
\text { observed }\end{array}$ & $\begin{array}{l}\text { FNA } \\
\text { performed }\end{array}$ \\
\hline Mediastinal (Naruke) & & & \\
1 & 6 & 0 & 0 \\
2-right & 16 & 3 & 2 \\
2-left & 6 & 3 & 1 \\
3 & 9 & 1 & 0 \\
4-right & 17 & 9 & 4 \\
4-left & 18 & 21 & 14 \\
5 & 13 & 25 & 8 \\
6 & 4 & 1 & 0 \\
7 & 37 & 56 & 48 \\
8-right & 7 & 10 & 4 \\
8-left & 1 & 7 & 5 \\
9-right & 2 & 2 & 1 \\
9-left & 1 & 2 & 1 \\
Celiac & 5 & 4 & 3 \\
Left adrenal & 10 & 8 & 7 \\
\hline & & & \\
\hline
\end{tabular}

mediastinal and/or upper retroperitoneal PET hot spots, mostly at Naruke level 4-right, 4-left, and 7 (table 2).

\section{EUS-FNA}

EUS-FNA was positive for malignancy in 50 patients, thereby avoiding subsequent surgical staging procedures in $62 \%$ of the patients (95\% CI 51 to 73 ). EUS-FNA was negative for malignancy in six (7\%) and inconclusive for malignancy in 25 (31\%) patients (fig 2). The accuracy of EUS-FNA was 0.77 (95\% CI 0.68 to 0.86 ). Most patients had EUS abnormalities 


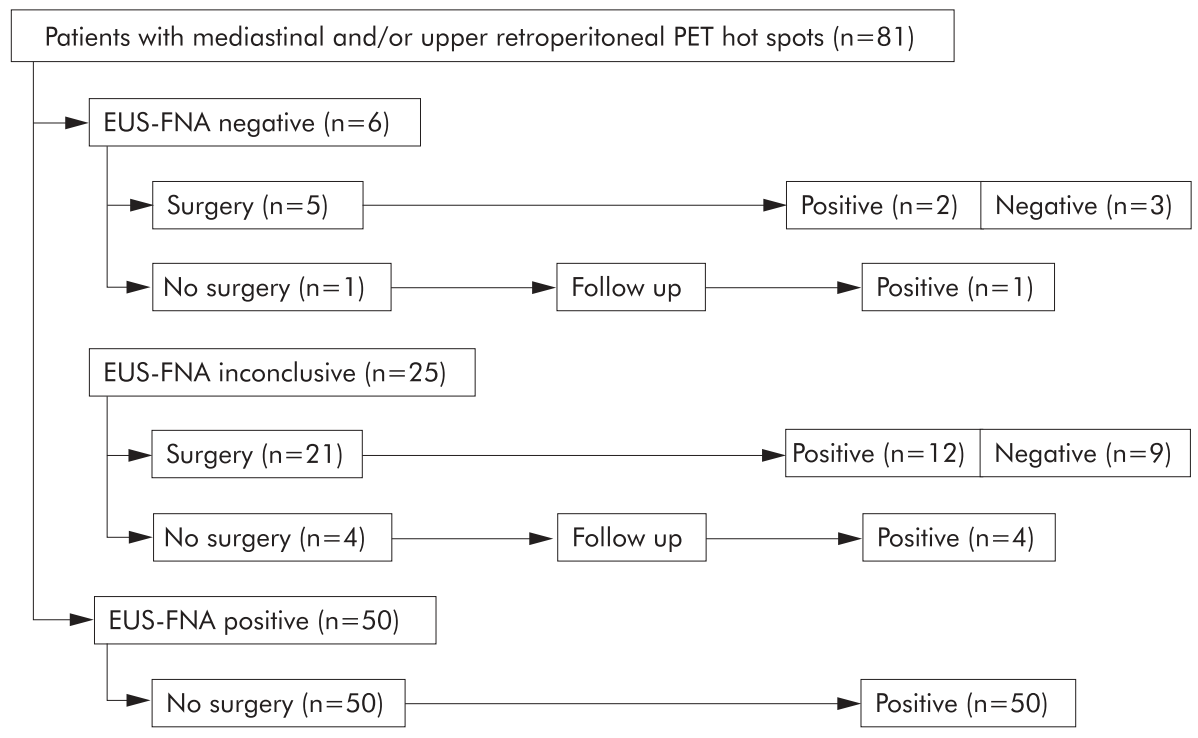

Figure 2 Flowchart of the enrolled patients.

at Naruke levels 4-left, 5, and 7 (table 2). After PET, EUS detected additional abnormalities at Naruke levels 4-left, 5, 7, 8-right, and 8-left. EUS was not able to visualise accurately Naruke levels 1, 2-right, 2-left, 3, or 4-right.

Twenty six of the 31 patients with a negative or inconclusive EUS-FNA underwent a surgical staging procedure: mediastinoscopy in 12 patients, mediastinotomy in one, exploratory thoracotomy with mediastinal dissection in nine, and mediastinoscopy followed by exploratory thoracotomy with mediastinal dissection in four patients. Of the six patients with a negative EUS-FNA, two had positive and three had negative mediastinal lymph nodes at surgery (fig 2). Further staging was not performed in one patient due to rapid progression of the mediastinal lesions. Of the 25 patients with an inconclusive EUS-FNA, 12 were positive and nine were negative for malignancy at surgery (fig 2). Four patients refused further surgical staging and, after follow up of 6 months, all had progressive disease diagnosed with imaging tests.

After all staging procedures including mediastinal dissection, mediastinal or upper retroperitoneal malignancy was not detected in 12 of the 81 patients (15\%). A negative or inconclusive EUS-FNA did not reliably prove the absence of mediastinal metastasis: 19 of the 31 patients $(68 \%)$ had lymph node metastasis at surgery. Of these 19 patients, seven had lymph node metastasis exclusively at the upper right mediastinum, outside the reach of EUS-FNA.
One mediastinal lymph node (which was localised at the PET hot spot) was randomly selected per patient for multiple logistic regression analysis. Lymph node size, shape (flat, round, irregular), boundary (vague, sharp), core structure (hyperechogenic, hypoechogenic), and visual impression (benign, malignant) were used as factors in the analysis. Lymph node size was the only significant predictor of malignancy $(p=0.03)$. For each additional centimetre the chance of malignancy increased by 0.56 (95\% CI 0.04 to 1.00).

The learning process of EUS-FNA of two residents was studied during 54 procedures, 29 by the first resident and 25 by the second. The numbers of lymph nodes detected by the residents failed to reach those identified by the experienced operators. More procedures are therefore required to learn EUS-FNA accurately by those who have not previously performed oesophageal endoscopy.

The median duration of the EUS-FNA procedure (including resident training) was 45 minutes (range 20-60). There was no morbidity or mortality during or after EUS-FNA.

\section{Economic analysis}

Costs are summarised in table 3. Hospitalisation was a major cost item for mediastinoscopy and exploratory thoracotomy with a mean (SE) duration of $3.0(0.3)$ days for mediastinoscopy and 12.0 (1.9) days for exploratory thoracotomy. Average staging costs were $\$ 2101$ per patient using a staging algorithm with EUS-FNA. For a staging algorithm without

Table 3 Real costs per procedure (US\$)

\begin{tabular}{lccc}
\hline Cost item & EUS-FNA & Mediastinoscopy & $\begin{array}{c}\text { Exploratory } \\
\text { thoracotomy }\end{array}$ \\
\hline Personnel & 92 & 308 & 517 \\
Equipment and materials & 498 & 143 & 400 \\
Medication & 2 & 28 & 91 \\
Overheads* & 208 & 168 & 352 \\
Other costs & 0 & 43 & 65 \\
Mean hospitalisation (days) & $10)$ & $(3)$ & $(12)$ \\
Hospitalisation (\$356/day) & 0 & 1068 & 4272 \\
ICU stay (days)† & $10)$ & $10)$ & $10.1)$ \\
ICU stay (\$1252/day)† & 0 & 0 & 125 \\
Total costs & 798 & 1758 & 5822 \\
\hline
\end{tabular}

*Calculated as $35 \%$ of the costs of personnel, equipment, materials, and medication.

tOne overnight stay in the intensive care unit (ICU) was assumed after $10 \%$ of the exploratory thoracotomies. 
EUS-FNA, average staging costs were \$3514 per patient, assuming that the ratio of mediastinoscopies to exploratory thoracotomies remained constant (17:13). Thus, mean cost savings through EUS-FNA were \$1413 (40\%) per patient. Sensitivity analyses were performed by varying the costs of the staging procedures. Cost savings could be maintained even if the costs of EUS-FNA were 2.5 times the current costs. Likewise, even with a $75 \%$ reduction in the costs of mediastinoscopy or a $50 \%$ reduction in the costs of exploratory thoracotomy, the outcome still favoured the EUS-FNA algorithm. Increasing the ratio of mediastinoscopies to exploratory thoracotomies from 17:13 to 10:1 in the staging algorithm without EUS-FNA (that is, a reduction in exploratory thoracotomies of $80 \%$ ) would result in equal costs of both staging algorithms.

\section{DISCUSSION}

Mediastinoscopy is still considered the gold standard for mediastinal staging of lung cancer. However, this procedure has no access to the aortopulmonary window and lower mediastinum, and has a complication risk of $2.5 \%{ }^{16}{ }^{17}$ EUSFNA, on the other hand, is a minimally invasive short outpatient procedure with hardly any complications. ${ }^{18}{ }^{19}$ It can reach aortopulmonary, subcarinal, and paraoesophageal lymph nodes, as well as the upper retroperitoneum. Due to air in the trachea, EUS cannot image the right upper mediastinum adequately. EUS-FNA is useful and appears more flexible than the transbronchial biopsy with a Wang needle. The nodes can be viewed directly and adjacent structures demonstrated. Mediastinoscopy and EUS-FNA may be considered as complementary techniques since their anatomical reaches are only partly overlapping. As shown in this study, EUS-FNA can limit the number of mediastinoscopies and exploratory thoracotomies in patients with irresectable tumours by $62 \%$. It is therefore the diagnostic test of first choice for further staging of lung cancer patients with mediastinal and/or upper retroperitoneal PET hot spots. ${ }^{20}$

Our report is the first study in which EUS-FNA has been used for cytological verification of PET hot spots in the mediastinum and upper retroperitoneum. Direct comparison of EUS-FNA with CT and PET is interesting, but it overlooks the actual clinical application of EUS-FNA. ${ }^{10}$ With a serial diagnostic approach, performing first CT and PET followed by EUS-FNA for cytological diagnosis, many surgical staging procedures will be prevented. The performance of EUS-FNA in patients with a PET negative mediastinum was not part of the study. However, some adenocarcinomas or other low grade tumours which may be PET negative and small lesions not resolving on PET may benefit from EUS-FNA. A randomised study will be needed to determine whether EUS-FNA assesses mediastinal metastases better than PET.

PET is not available in all hospitals. However, EUS-FNA can be guided by CT or even used as the first procedure after (or in conjunction with) bronchoscopy in lung cancer staging. EUS-FNA proved to be of great value in this respect. ${ }^{10}$

The decrease in surgical staging procedures in our study was higher than we initially expected. This can partly be explained by a high number of tertiary referrals, resulting in a higher positive predictive value of PET hot spots than expected $(85 \% \vee 67 \%)$. This figure is similar to the positive predictive values reported in a recent meta-analysis. ${ }^{5}$

Procedures that may be an alternative to EUS-FNA include percutaneous transthoracic needle biopsy (PTNB), transbronchial fine needle aspiration (TBNA), and endobronchial ultrasonography (EBUS). As outlined in a recent review by our study group, PTNB cannot reach as many lymph node stations as EUS-FNA and it has a higher complication rate. The performance of TBNA in mediastinal lymph node staging is variable, and it is only possible for lymph nodes neighbouring large airways. ${ }^{20}$ The use of EBUS is promising but there is still little experience with this technique. ${ }^{21}$

Diseases other than lung cancer can also be identified with EUS-FNA. Diagnosis and staging are usually performed in concert, since the most efficient way to make a diagnosis is often dictated by the stage of the cancer. ${ }^{22}$ We encountered $13 \%$ of patients with other diagnoses such as malignant lymphoma, sarcoidosis, and aspergillosis. They mostly presented with atypical signs, symptoms, and CT abnormalities, and without a pathological diagnosis.

The resolution of EUS-FNA within a view field of several centimetres is higher than that of PET, and at least that of CT. ${ }^{23-25}$ Abnormalities of 2-3 mm can easily be located and punctured. EUS-FNA can also localise mediastinal tumour invasion. Moreover, it can be used to search for metastases in areas surrounding mediastinal invasion by tumour, thereby selecting patients who are candidates for more extensive surgery. The number of inconclusive cytological results will increase, since more normal lymph nodes are punctured.

In this study the ability of EUS to identify lymph nodes at the site of PET hot spots was total, but FNA punctures were inconclusive in almost one third of the patients. This can partly be explained by the bloody content of the EUS-FNA specimens and sometimes by attempts to puncture very small lymph nodes. Moreover, in most cases after three punctures at the same site no further punctures were performed. Replacing the on-site cytotechnician by an on-site cytopathologist may perhaps increase the number of adequate specimens obtained. ${ }^{26}$

We analysed the number of procedures required to learn accurate EUS-FNA and found that less than 30 procedures are not sufficient to acquire the necessary skills. We estimate that about 40 procedures will be enough to perform EUSFNA adequately, including interpretation of ultrasound images of the left adrenal glands and mediastinal lymph node stations.

The cost effectiveness of EUS-FNA in this study was not based on a decision analysis model but on real cost assessments. The addition of EUS-FNA to the staging algorithm avoided surgical staging procedures in $62 \%$ of patients, thereby decreasing staging costs by $40 \%$. Our sensitivity analyses show that the cost effectiveness of EUSFNA is maintained over a wide variation in procedure costs.

In conclusion, EUS-FNA is a safe and cost effective procedure for lung cancer staging which detects additional mediastinal abnormalities after PET.

\section{ACKNOWLEDGEMENTS}

The authors thank C A van Minnen, pulmonary physician, for his critical review of the manuscript and A A Smidt, endoscopy nurse, for her extensive support during the EUS-FNA procedures.

\section{Authors' affiliations}

H Kramer, J W G van Putten, H J M Groen, Department of Pulmonary Diseases, Groningen University Hospital, Groningen, The Netherlands H M van Dullemen, Department of Gastroenterology, Groningen University Hospital, Groningen, The Netherlands

A H H Bongaerts, Department of Radiology, Groningen University Hospital, Groningen, The Netherlands

A J H Suurmeijer, Department of Pathology and Laboratory Medicine, Groningen University Hospital, Groningen, The Netherlands

T J Klinkenberg, Department of Thoracic Surgery, Groningen University Hospital, Groningen, The Netherlands

W J Post, H Groen, Office for Medical Technology Assessment, Groningen University Hospital, Groningen, The Netherlands J Pruim, Positron Emission Tomography Centre, Groningen University Hospital, Groningen, The Netherlands

This study was supported by the Netherlands Organization for Health Research and Development through research grant \#945-10-003. 


\section{REFERENCES}

1 Mountain CF. Revisions in the international system for staging lung cancer. Chest 1997; 111:1710-7.

2 Mountain CF, Dresler CM. Regional lymph node classification for lung cance staging. Chest 1997;111:1718-23.

3 Robinson LA, Wagner H Jr, Ruckdeschel JC. Treatment of stage IIIA non-small cell lung cancer. Chest 2003; 123:202-20S.

4 Dwamena BA, Sonnad SS, Angobaldo JO, et al. Metastases from non-small cell lung cancer: mediastinal staging in the 1990s: meta-analytic comparison of PET and CT. Radiology 1999:213:530-6.

5 Hellwig D, Ukena D, Paulsen F, et al. Meta-analysis of the efficacy of positron emission tomography with F-18- fluorodeoxyglucose. Pneumologie 2001;55:367-77.

6 Pieterman RM, Van Putten JWG, Meuzelaar JJ, et al. Preoperative staging of non-small-cell lung cancer with positron-emission tomography. N Engl J Med 2000;343:254-61.

7 Vansteenkiste JF, Stroobants SG, De Leyn PR, et al. Lymph node staging in non-small-cell lung cancer with FDG-PET scan: a prospective study on 690 lymph node stations from 68 patients. J Clin Oncol 1998; 16:2142-9.

8 Lloyd C, Silvestri GA. Mediastinal staging of non-small-cell lung cancer. Cancer Control 2001;8:311-7.

9 Wiersema MJ, Kochman ML, Cramer HM, et al. Preoperative staging of non-small cell lung cancer: transesophageal US-guided fine-needle aspiration biopsy of mediastinal lymph nodes. Radiology 1994; 190:239-42.

10 Fritscher-Ravens A, Bohuslavizki KH, Brandt L, et al. Mediastinal lymph node involvement in potentially resectable lung cancer: comparison of CT, positron emission tomography, and endoscopic ultrasonography with and without fineneedle aspiration. Chest 2003;123:442-51.

11 Chang KJ, Erickson RA, Nguyen P. Endoscopic ultrasound (EUS) and EUSguided fine-needle aspiration of the left adrenal gland. Gastrointest Endosc 1996:44:568-72

12 Wiersema MJ, Hassig WM, Hawes RH, et al. Mediastinal lymph node detection with endosonography. Gastrointest Endosc 1993;39:788-93.
13 Oostenbrink JB, Koopmanschap MA, Rutten FFH. Handbook for cost studies, methods and guidelines for economic evaluation in health care. Amstelveen: College for Health Insurances, 2000.

14 Gold MR, Siegel JE, Russell LB, et al. Cost-effectiveness in health and medicine. New York: Oxford University Press, 1996.

15 Drummond MF, O'Brien BJ, Stoddart GL, et al. Methods for the economic evaluation of health care programmes, 1st ed. New York: Oxford University Press, 1998.

16 Bogot NR, Shaham D. Semi-invasive and invasive procedures for the diagnosis and staging of lung cancer. II. Bronchoscopic and surgical procedures. Radiol Clin North Am 2000;38:535-44.

17 Mentzer SJ, Swanson SJ, DeCamp MM, et al. Mediastinoscopy, thoracoscopy, and video-assisted thoracic surgery in the diagnosis and staging of lung cancer. Chest 1997;112:239-41S

18 Barawi M, Gottlieb K, Cunha B, et al. A prospective evaluation of the incidence of bacteremia associated with EUS-guided fine-needle aspiration. Gastrointest Endosc 2001;53:189-92.

19 Affi A, Vazquez-Sequeiros E, Norton ID, et al. Acute extraluminal hemorrhage associated with EUS-guided fine needle aspiration: frequency and clinical significance. Gastrointest Endosc 2001;53:221-5.

20 Kramer H, Groen HJM. Current concepts in the mediastinal lymph node staging of nonsmall cell lung cancer. Ann Surg 2003;238:180-8.

21 Okamoto H, Watanabe K, Nagatomo A, et al. Endobronchial ultrasonography for mediastinal and hilar lymph node metastases of lung cancer. Chest 2002;121:1498-506.

22 Rivera MP, Detterbeck FC, Mehta AC. Diagnosis of lung cancer: the guidelines. Chest 2003;123:129-36S

23 Tarantola G, Zito F, Gerundini P. PET instrumentation and reconstruction algorithms in whole-body applications. J Nucl Med 2003:44:756-69.

24 Fritscher-Ravens A. Endoscopic ultrasound evaluation in the diagnosis and staging of lung cancer. Lung Cancer 2003;41:259-67.

25 Fuchs T, Kachelriess M, Kalender WA. Technical advances in multi-slice spiral CT. Eur J Radiol 2000;36:69-73.

26 Klapman JB, Logrono R, Dye CE, et al. Clinical impact of on-site cytopathology interpretation on endoscopic ultrasound-guided fine needle aspiration. Am J Gastroenterol 2003;98:1289-94.

\section{LUNG ALERT}

\section{A novel method for the diagnosis of lung transplant rejection}

$\Delta$ Aharinejad S, Taghavi S, Klepetko W, Abraham D. Prediction of lung-transplant rejection by hepatocyte growth factor. Lancet 2004;363:1503-8

$\mathrm{T}$

he most common causes of morbidity and mortality following lung transplantation are primary graft failure, infection, and acute and chronic rejection. Clinical criteria alone are inaccurate for the diagnosis of graft rejection. The diagnosis can be made by transbronchial biopsy but this procedure is invasive and cannot be performed repeatedly. Hepatocyte growth factor (HGF) is a glycoprotein highly expressed in the lung parenchyma and produced after acute lung injury. This study was designed to assess whether serum HGF could act as a marker of acute rejection following lung transplantation.

The authors measured serum concentrations of HGF by ELISA in 109 patients undergoing lung transplantation ( 65 with chronic obstructive pulmonary disease, 23 with cystic fibrosis, and 21 with idiopathic pulmonary fibrosis) and 12 healthy controls. The mean serum HGF concentration before transplantation was higher in the patients than in the controls, and postoperatively rose significantly on the first day before decreasing by day 3 . At an episode of acute rejection the serum HGF concentration was significantly higher than in all other groups (controls, patients before transplantation, patients with no rejection, and patients with infection). Logistic regression analysis identified the serum HGF concentration as an independent predictor of lung graft rejection $(p=0.012)$. After steroid treatment HGF concentrations returned to the preoperative value within 3 days.

The authors conclude that, since serum concentrations of HGF increase with graft rejection but not infection and the values decline with steroid treatment, serum HGF monitoring could be a sensitive predictor of lung graft rejection.

R Agarwal

Senior Resident, Department of Pulmonary Medicine, Postgraduate Institute of Medical Education and Research, Chandigarh, India drritesh1@rediffmail.com 